\title{
Polarities of G. Higman's symmetric design and a strongly regular graph on 176 vertices
}

\author{
A. E. BRouWER
}

\begin{abstract}
We investigate the polarities of G. Higman's symmetric $2-(176,50,14)$ design and find that there are two of them (up to conjugacy), one having 80 and the other 176 absolute points. From the latter we can derive a strongly regular graph with parameters $(v, k, \lambda, \mu)=(176,49,12,14)$. Its group of automorphisms is Sym(8) with orbits of size 8 and 168 on the vertices. It does not carry a partial geometry or a delta space, and is not the result of merging $d=1$ and $d=2$ in a distance regular graph with diameter 3 and girth 6 on 176 vertices.
\end{abstract}

\section{Introduction}

It is well-known that given a symmetric $2-(V, K, \Lambda)$ design with a polarity, we can find a strongly regular graph with parameters $(v, k, \lambda, \mu)=(V, K, \Lambda, \Lambda)$ if the polarity has no absolute points, and one with parameters $(V, K-1, \Lambda-2, A)$ if all points are absolute. (In the first case take the symmetric incidence matrix of the design as adjacency matrix of the graph; in the second case first delete the diagonal.)

In [4] G. Higman constructs a symmetric 2-(176,50,14) design with the Higman-Sims group as group of automorphisms, and indicates a polarity. Using the description given in Sims [6] it is trivial to check that the polarity has only absolute points (but note that Sims's statement about the orbit lengths is inaccurate) so that we get a $(176,49,12,14)$ graph.

Computing the multiplicities of the eigenvalues shows that no $(176,50,14,14)$ graph can exist, i.e., any polarity of the design must have absolute points. In fact,

AMS (1980) subject classification: Primary 05C25, 20B25, $20 \mathrm{D} 08$.

Manuscript received August 26, 1982. 
the number of nonabsolute points is divisible by 12 , so that the number of absolute points is congruent $8(\bmod 12)$. Below we study this graph in some more detail. Looking at the possible polarities of the symmetric design we find that up to conjugation there is only one other polarity - one with 80 absolute points. Consequently our graph is the only one that can be derived in this way from the symmetric design.

\section{G. Higman's symmetric design}

C. C. Sims [6] gives the following description of a symmetric $2-(176,50,14)$ design: There are six ways to make a set of six elements into a projective line, or, equivalently there are six transitive representations of $\operatorname{PGL}(2,5)$ on six given points. Now fix a set $B$ of eight elements and let $C$ be the set of $168=6 \cdot\left(\begin{array}{l}8 \\ 2\end{array}\right)$ groups PGL $(2,5)$ acting on $B$ with orbits of sizes $1,1,6$. Both the points and the blocks of the design will be indexed by $B \cup C$. If $x \in B \cup C$ then let $x$ denote a point of the design and $L_{x}$ a block of the design; $\pi: x \mapsto L_{x}$ will be a polarity of the design. Incidences are defined as follows:

for $b \in B: L_{b}=B \cup\{H \in C \mid H$ fixes $b\}$,

for $H \in C: L_{H}=\{b \in B \mid H$ fixes $b\}$

$\cup\left\{G \in C \mid H\right.$ has less than 60 conjugates under $\left.N_{\text {sym(8) }}(G)\right\}$.

Since the 168 elements of $C$ fall into orbits of sizes $1+5+(6+6)+30+(30+30)+$ 60 under conjugation with elements of $G$ (where the bracketed orbits fuse under $N(G))$ we see that $\left|L_{b}\right|=8+42=50$ and $\left|L_{H}\right|=2+1+5+12+30=50$, so that blocks have size 50. It is an easy (but somewhat tedious) exercise to check that $\lambda=14$. [It might be useful to notice that the orbits can be described as follows:

* $\quad$ 1: $G=H$;

* 5: $G$ and $H$ have same fixed points but are not identical;

* 6+6: $G$ and $H$ have one fixed point in common and $|G \cap H|=20$ (i.e., $G \cap H$ contains an element of order 5);

30+30: $G$ and $H$ have one fixed point in common and $|G \cap H|=4$

30: $G$ and $H$ have no fixed points in common and $|G \cap H|=4$ (i.e., $G \cap H$ contains an element of order 4 );

60: $G$ and $H$ have no fixed points in common and $|G \cap H|=2$.

Here the asterisks mark the cases where $G \in L_{H}$ or, equivalently $H \in L_{G}$. So, obviously, $x \mapsto L_{x}$ indeed is a polarity.]

The automorphism group of this design is the simple Higman-Sims group HS. It

]. a

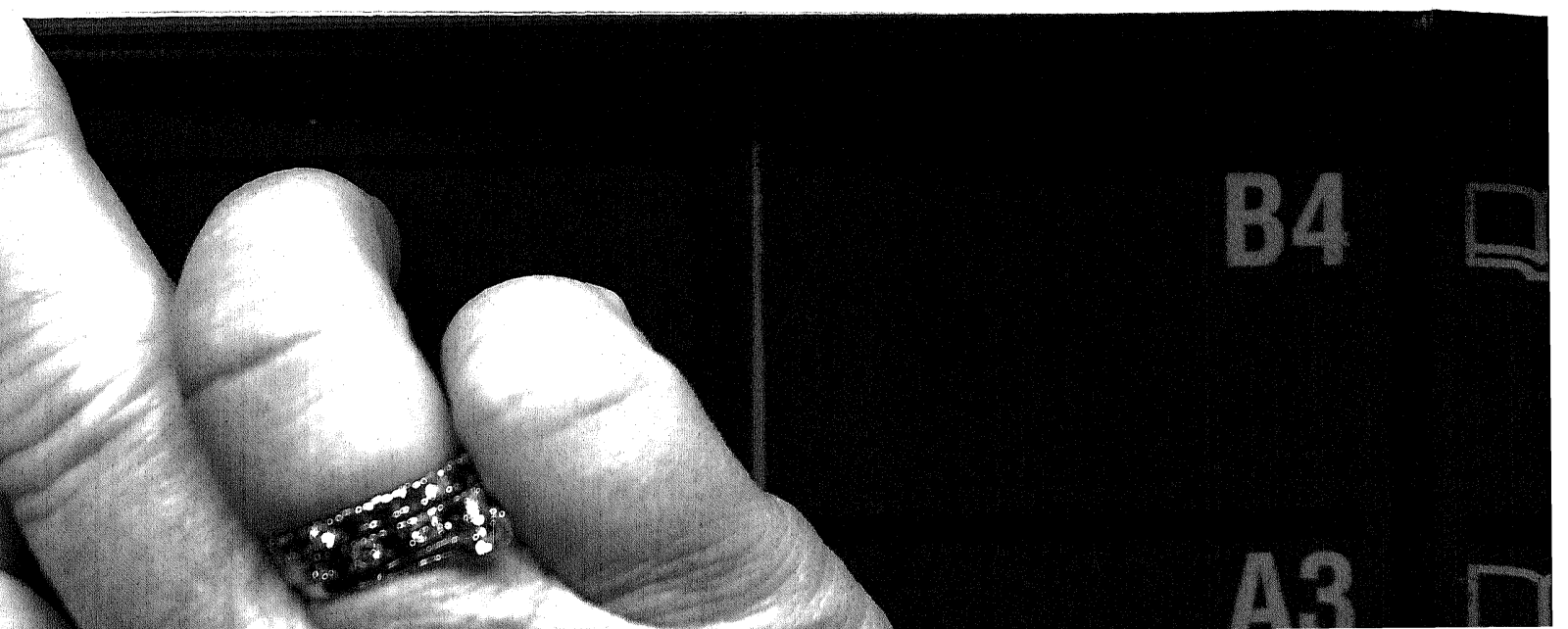


has index 2 in the group of correlations $\langle\mathrm{HS}, \pi\rangle \cong$ Aut HS. From the description given it is clear that $\operatorname{Sym}(8)$ centralizes $\pi$; in fact $C_{\mathrm{HS}}(\pi) \cong \operatorname{Sym}(8)$. Consequently, if $\Gamma$ is the graph derived from the design with polarity $\pi$ then Aut $\Gamma \equiv \operatorname{Sym}(8)$, and Aut $\Gamma$ has orbits $B$ and $C$ of sizes $8+168$ on the vertices of $\Gamma$.

\section{Polarities of the design}

From the character table (see Frame [2]) we see that there are two conjugacy classes of involutions in (Aut HS) $\backslash$ HS. One is the above polarity with 176 absolute points; we want to find the number of absolute points under the other polarity. To do this, consider the permutation representation of Aut HS on the unordered flags, and compute the corresponding character $\chi$. For the classes of involutions $C_{1}^{\prime}$ and $C_{10}^{\prime}$ (in Frame's notation) we find $\chi\left(C_{1}^{\prime}\right)=176$ and $\chi\left(C_{10}^{\prime}\right)=80$ so that the other polarity has only 80 absolute points and cannot be used to construct a strongly regular graph. [As follows: first identify the elements of Sym(7), the stabilizer of a flag in HS, among the conjugacy classes of HS. In fact we can identify the elements of $\operatorname{Sym}(8)$ as follows:

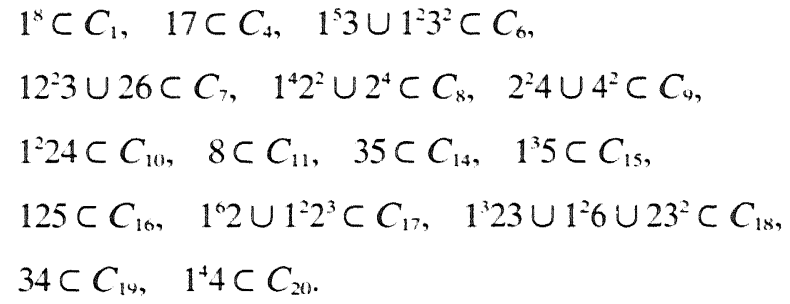

Next observe that $\chi$ is induced by 1 on $\operatorname{Sym}(7)$, so that we find the decomposition of $\chi$ in HS by Frobenius reciprocity. This yields (in HS)

$$
\chi=1+77+3 * 175+231+2 * 693+154+1386+2 * 2520
$$

so that $\chi$ has values

$$
(8800,0,1,0,25,1,160,0,8,0,0,0,30,2,72,9,1,160,0,0,0)
$$

on the classes of HS (in the order given by Frame). The requirement that $\chi$ has nonnegative values now determines $\chi$ on the classes of (Aut HS) $\backslash$ HS. We find the decomposition (in Aut HS)

$$
\chi=1+77+2 * 175+\overline{175}+\overline{231}+2 * 693+154+\overline{1386}+2520+\overline{2520},
$$


and the values

$(176,1,5,1,16,0,0,0,0,80,5,5,0,6,0,0,0,0)$

on the classes of (AutHS) $\backslash$ HS. Here the bar above a character denotes the associate character (i.e., the negative of the column given by Frame). The computations were checked by explicit computation of $\chi$ on the classes of $\operatorname{Sym}(8)$.]

We can describe the polarities explicitly in several ways.

A. In Sims' representation, the elements $1^{8}$ and $1^{6} 2$ of $\operatorname{Sym}(8)$ give a polarity with 176 absolute points, while $1^{4} 2^{2}$ and $1^{2} 2^{3}$ give polarities with 80 absolute points.

B. Margaret Smith [7] has given a beautiful description of the symmetric design, as follows: Let $u, v$ be two fixed points of the Steiner system $\mathrm{S}(5,8,24)$. Let the points of the design be the blocks of the Steiner system containing $u$ but not $v$. Let the blocks of the design be the blocks of the Steiner system containing $v$ but not $u$. Define incidence between points and blocks by $B \sim B^{\prime}$ iff $\left|B \cap B^{\prime}\right| \in\{0,4\}$. Using the parameters of the near-hexagon associated with $S(5,8,24)$ one immediately sees that this defines a design with the right parameters.

From Conway [1] we learn an explicit representation of $\mathrm{S}(5,8,24)$ showing large parts of its group of automorphisms. In fact, consider $4 \times 6$ matrices with entries 0 or 1 satisfying the following restraints:

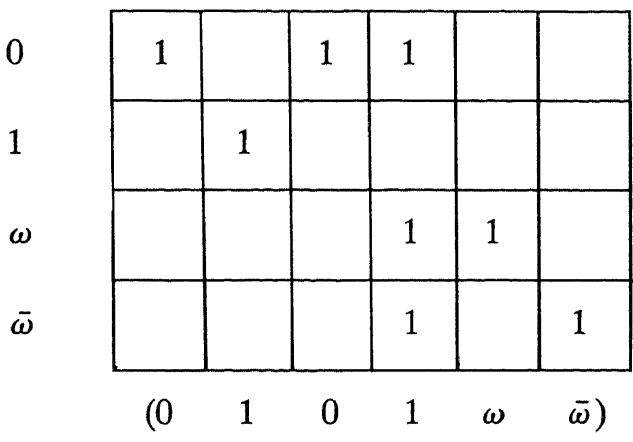

(i) the six column sums and the sum of the first row have the same parity;

(ii) if $r_{i}$ denotes the $i$ th row $(1 \leqq i \leqq 4)$ and $\mathbb{F}_{4}=\{0,1, \omega, \bar{\omega}\}$ and $C$ is the linear $[n, k, d]=[6,3,4]$ code over $\mathbb{F}_{4}$ generated by the rows of the matrix

$$
\left(\begin{array}{llllll}
1 & 0 & 0 & 1 & \omega & \bar{\omega} \\
0 & 1 & 0 & 1 & \bar{\omega} & \omega \\
0 & 0 & 1 & 1 & 1 & 1
\end{array}\right)
$$

then $r_{2}+\omega r_{3}+\bar{\omega} r_{4} \in C$.

It is almost trivial to verify that these matrices form a linear $[24,12,8]$ code over $\mathbb{F}_{2}$, i.e., the extended Golay code. The Steiner system $S(5,8,24)$ is obtained by taking the matrices with weight 8 . 
Now, if $\sigma$ is an involution in $M_{24}$ interchanging $u$ and $v$ (say $u=(1,5$ ), $v=(1,6)$ ), then $\sigma$ defines a polarity of the design. The involutions with 8 fixed points (such as: interchange the last two rows and the last two columns) have 176 absolute points, and those without fixed points (such as: interchange 1st and 2nd, 3 rd and 4th, 5th and 6th column, and 2 nd and 3rd row) have 80 absolute points.

\section{The structure of the graph $\Gamma$}

Let $\Gamma$ be the $(176,49,12,14)$ strongly regular graph constructed above. By choosing one fixed polarity we destroyed much of the symmetry in the design - the graph $\Gamma$ is a rather ugly-looking animal. As we saw above, its group is $\operatorname{Sym}(8)$ and has orbits of sizes 8 and 168 on the vertices. Since the graph has the right parameters for the point graph of a partial geometry $\operatorname{pg}(8,7,2)$, we investigate its structure somewhat more closely. Let a line be an 8-clique.

Each point $b \in B$ is in 8 lines, forming the 'comb' configuration in $\Gamma_{b}$ :

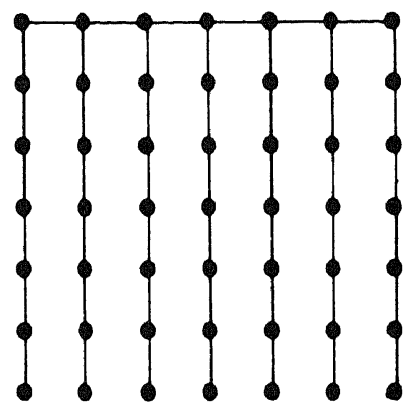

In fact, $\Gamma_{b}$ can be obtained from the Hoffman-Singleton graph by deleting a vertex $x_{0}$, making its set of neighbours $\left\{x_{1}, \cdots, x_{7}\right\}$ into a 7 -clique, and then making the set of neighbours of $x_{i}$ together with $x_{i}$ itself into a 7-clique for $1 \leq i \leq 7$.

Each point $c \in C$ is in 6 lines, forming a comb with two teeth missing:

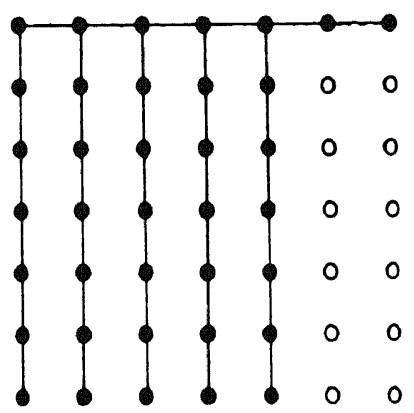


$\Gamma_{c}$ contains the following maximal cliques: $18 * K_{2}, 50 * K_{3}$ and $6 * K_{7}$. It can be obtained from the Hoffman-Singleton graph by deleting a vertex $x_{0}$, making its set of neighbours $\left\{x_{1}, \cdots, x_{7}\right\}$ into a 7 -clique, next making the set of neighbours of $x_{i}$ together with $x_{i}$ itself into a 7 -clique for $1 \leqq i \leqq 5$, and finally putting a $K_{6,6}$ between the neighbours of $x_{0}$ and those of $x_{7}$. We see that 12 of the neighbours of $c$ are not in 7-cliques, i.e., 12 of the edges incident with $c$ are not in a line, and the graph does not carry a partial geometry.

The complementary graph $\bar{\Gamma}$ satisfies the parameter restrictions for delta-spaces $\Delta(3,63,35,45)$ or $\Delta(4,42,35,30)$. But if $a b$ is an edge of $\Gamma$ contained in two lines, then the subgraph of $\bar{\Gamma}$ consisting of the points non-adjacent to both $a$ and $b$ is the complete bipartite graph $K_{6,6}$ and does not contain cliques of size 3 or 4 . Hence $\bar{\Gamma}$ does not carry a nontrivial delta-space.

It is not inconceivable that a distance regular graph with diagram

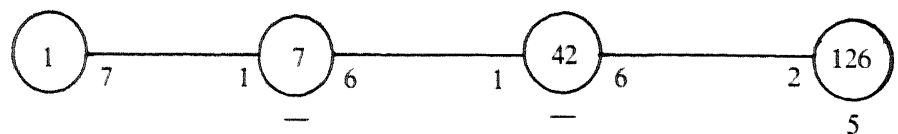

exists. The 'distance 3' graph has the parameters of $\bar{\Gamma}$, and so one might hope to obtain such a distance regular graph by choosing 7 of the 49 edges incident with each vertex of $\Gamma$. However, this is not possible: in such a graph the 'stars' would be 8-cliques of $\Gamma$, and the 8 stars on a fixed point would always form the picture of $\Gamma_{b}$, never that of $\Gamma_{c}$ above.

\section{REFERENCES}

[1] Conway, J. H., Oral communication by J. H. van Lint. Oct. 1980.

[2] FRAME, J. S., Computation of characters of the Higman-Sims group and its automorphism group. J. Algebra 20 (1972), 320-349.

[3] Higman, D. G. and Sims, C. C., A simple group of order 44,352,000. Math. Z. 105 (1968), 110-113.

[4] Higman, G., On the simple group of D. G. Higman and C. C. Sims. Illinois J. Math. 13 (1969), 74-80.

[5] Magliveras, S. S., The subgroup structure of the Higman-Sims simple group. Bull. Amer. Math. Soc. 77 (1971), 535-539.

[6] Sims, C. C., On the isomorphism of two groups of order 44,352,000. In Theory of Finite Groups (Symposium, Harvard Univ., Cambridge, 1968), Benjamin, New York, 1969, 101-108.

[7] SMITH, M. S., On the isomorphism of two simple groups of order 44,352,000. J. Algebra 41 (1976), $172-174$.

[8] SMrth, M. S., A combinatorial configuration associated with the Higman-Sims simple group. J. Algebra 41 (1976), 175-195.

Math. Centre,

Kruislaan 413,

NL-1098SJ Amsterdam,

The Netherlands 